\section{In a spin}

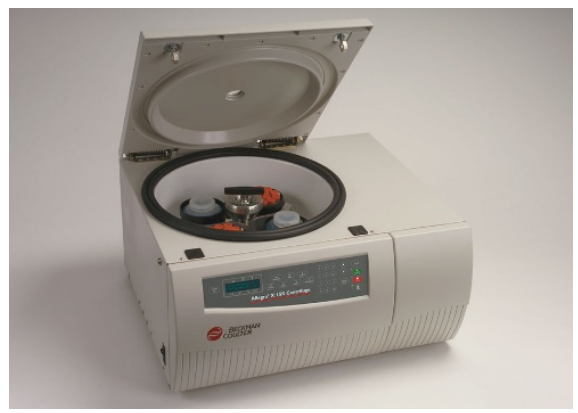

Allegra $\mathrm{X}-15 \mathrm{R}$ for spins up to $5,250 \mathrm{~g}$.

Beckman Coulter introduces the Allegra X-15R benchtop centrifuge, providing fast separations and optimal recovery in the large capacity $4 \times 750-\mathrm{ml}$ rotor. This new instrument spins the popular rotor at $5,250 \mathrm{~g}$ and also spins microplate carriers. Suitable for many applications, the Allegra X-15R accommodates Beckman Coulter's exclusive adapters for direct centrifugation of cell culture flasks, eliminating an entire step in the cell culture process. It also incorporates ARIES Smart Balance Rotor technology, which not only automatically detects imbalance but also corrects it, enabling customers to complete their runs even if the samples are not balanced. Aerosolve clear canisters for the round swinging buckets are also available for biocontainment, enabling viewing from top and bottom. An easy-to-use numeric keypad user interface enables direct entry of values with user-friendly prompts.

http://www.beckmancoulter.com

Tel. (+1) 800-742-2345 (US)/

(+1) 714-871-4848 (elsewhere)

\section{Off the shelf}

Apotech's latest edition of ApoNews highlights new tools for endoplasmic reticulum (ER) stress-mediated apoptosis. Also highlighted are a number of new TNF superfamily and granzyme A-related antibodies for inflammation and adiponectin/ACRP30 research. The new antibodies for studying stress-related apoptosis include antibodies to ATF6, BAP31, caspase-12, CHOP, TRAF2 and BiP/GRP78. There are also new TLR ligands for inflammation research and monoclonal antibodies to HSP70, HSP90, HVEM, LTBR, osteoprotegerin and acid nuclear phosphoprotein 32A. http://www.apotech.com

Tel. (+1) 800-958-9007 (US)/

(+1) 858-550-8828
Pure and simple

Qbiogene has introduced three new kits for purifying mRNA directly from cells, tissues or total RNA. The kits have been formulated to give higher yields than oligo-dT-based purification techniques. mTRAP mRNA purification kits incorporate a peptide nucleic acid molecular probe that has a high binding affinity for mRNA. As a result, mRNA isolation can be performed in buffers with lower salt concentrations, eliminating secondary structure that can make mRNA inaccessible for capture, as well as non-specific bonds that can cause unwanted rRNA and protein contamination. The system also allows mRNA to be selectively eluted away from unwanted genomic DNA and makes mRNA with short poly-A tails more available for capture. mTRAP kits are available in two sizes for purification directly from tissue or cells and in one size for the purification of mRNA from total RNA. The 24-prep mTRAP mini kit is said typically to yield $10-20 \mu \mathrm{g}$ of mRNA from $0.5-1.0 \times 107$ cells pr 50-200 mg tissue, whereas the 6-prep mTRAP maxi kit typically yields $100-200 \mu \mathrm{g}$ of mRNA of mRNA from $0.5-2.0 \times 108$ cells or $0.4-1.0 \mathrm{~g}$ tissue. mRNA yields from the 12-prep mTRAP total kit are typically $10-15 \mu \mathrm{g}$ from each $500 \mu \mathrm{g}$ of total RNA. All three kits include the required plasticware and Rnasefree solutions.

http://www.qbiogene.com

Tel. (+1) 800-424-6101 (US)/

(+1) 760-929-1700

\section{Motor coordination assessment}

The Rota-Rod treadmill for rats and mice from Stoelting is designed to accurately test motor coordination and fatigue in rodents. Consequently, researchers are able to measure the effects of drugs, brain damage or disease on animal behavior. Interchangeable drums are available for mice, rats or both. Researchers can set up the Rota-Rod at either constant speed or a gradual acceleration, controlled by a precise microprocessor and displayed on a clear LCD panel. All necessary software is included with the treadmill and allows researchers to transfer data onto a PC.

http://www.stoeltingco.com/physio Tel. (+1) 630-860-9700

\section{Buffers}

The siPORT siRNA electroporation buffer from Ambion is intended for delivery of primary and difficult-to-transfect cells with siRNAs. The buffer can be used with multiple types of electroporators to ensure highly efficient siRNA delivery into cells while maintaining high cell viability.

http://www.ambion.com

Tel. (+1) 800-888-8804 (US)/

(+1) 512-651-0200

\section{Concentrators}

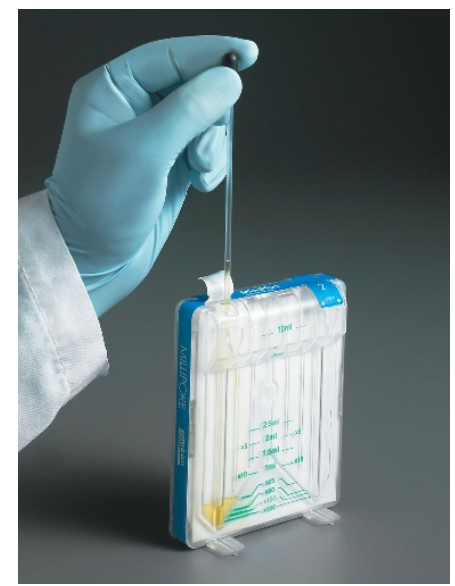

Millipore's answer to concentrating and desalting proteins.

Millipore offers Minicon and Miniplus concentrators for concentrating and desalting proteins in urine or CSF samples. The devices allow users to rapidly and conveniently concentrate proteins, including Bence Jones proteins, which are used to diagnose and monitor multiple myeloma, a tumor of plasma cells. The easy-to-use Minicon and Miniplus concentrators consist of individual cells containing an ultrafiltration membrane backed by a pad that absorbs water and solutes from the sample. Proteins retained by the membrane are concentrated, desalted, and ready for subsequent analysis by electrophoresis. Depending on the model, Minicon devices can simultaneously concentrate 9 or 10 samples to $100 \mathrm{x}$ in 80 to $120 \mathrm{~min}$. The Miniplus device can concentrate 4 samples to $200 \mathrm{x}$ in 3 to $4 \mathrm{~h}$. Available with membranes in a variety of molecular weight cut-offs, the devices have a maximum sample volume of $10 \mathrm{ml}$ and a minimum recovery volume from 30 to $50 \mu \mathrm{l}$. http://www.millipore.com/clinical Tel. (+1) 1-800-MILLIPORE 